\title{
MODELING AND SIMULATION IN DEFENSE AND SECURITY NATO CONTEXT
}

\begin{abstract}
M odeling and Simulation $(\mathrm{M} \& S)$ are not new phenomena in the world of the military. Military training has always involved certain ways of simulating the reality of the battlefield. Depending on the level at which simulation was done it also involved more or less sophisticated modeling activities. The simulations could cover various aspects such as maneuvers, weapons used, own weapons as well as those the opponent was supposed to use and so on. The possibilities of modeling and simulation in the training area became well known after the Great explosion in the computer technology. Now the second stage in Modeling and Simulation is presented and this is the use of the Net for simulation.
\end{abstract}

Still, a policy for developing and using modeling and simulation all over the Euroatlantic Alliance has not been developed yet. For a number of years nations continued their own policies, in more or less isolated manner, and cooperation was limited to some specific technological domains. As a broad subject, simulation was first introduced on the international scene as a Common European Priority Area (CEPA) within the EUCLID program, then part of the IEPG, later part of the WEU WEAG activities. In the NATO context simulation came up again as a special topic in a symposium on this subject, organized by the US for the NATO DRG in September 1995. Clearly there was coordination, but not in the sense of a focussed effort linking modeling and simulation together.

\section{NATO Involvement}

The growing importance of modeling and simulation was recognized by NATO through the establishment of a special Steering Group on Simulation Policy and Applications (SGMS) by the Conference of National Armament Directors in $1996 .{ }^{1}$ This group was to carry on earlier work by a special expert group. The SGMS was charged in particular with the task of formulating a NATO policy on this subject, in particular including a Master Plan that would be the guide to get deeper involvement by NATO nations and NATO itself. Of particular importance was the issue that the 
various national developments should be guided in such a way that interoperability between various modeling and simulation systems would be assured to the maximum extent possible. This seemed all the more important as simulation, as a result of the rapid spread of information technologies, is more and more used on national levels. For the Alliance, the integration of national assets into Allied Command Structures is of prime importance. The national modeling and simulation developments and capabilities should take this into account and make sure that simulation and training activities can be conducted at allied levels.

Given the many technological aspects of M\&S and the earlier involvement of DGR groups in this subject, the CNAD decided to have the SGMS report to it through the new Research and Technology Board (RTB) that was officially inaugurated in November 1996. The SGMS did report its progress to the RTB during its meetings and in September 1998 the final report of the SGMS was presented to the RTB at its meeting in Athens.

The SGMS had made tremendous progress in the time allotted to it. The acceptance by the whole SGMS of High Level Architecture (HLA) as the common baseline could be called a small miracle. Proposals for other activities to forward the M\&S work are all of good quality. In organizational terms, the very nature of $M \& S$ makes it necessary to embed follow-on activity with the military user, the acquisition community, the C3 community and the world of research and technology. It is therefore not surprising that ultimately the CNAD, the Military Committee and finally the North Atlantic Council opted for the attachment of the follow-on work to the Research \& Technology Organization (RTO). The NATO Modeling and Simulation Group and the supportive staff of the Modeling and Simulation Co-ordination Office have found their place in the RTO. With the first NATO/PfP Conference on Modeling and Simulation in the fall of 1999 in Norfolk, VA, the RTB really started focusing on the M\&S program.

\section{Vision Statement}

Modeling and simulation will provide a readily available, flexible and cost-effective means to enhance NATO operations dramatically in the application areas of defense planning, training, exercises, support to operations, research, technology development and armaments acquisition. This goal will be supported by Bulgarian MoD effort that promotes interoperability, reuse and affordability.

\section{Guiding Principles}

Based on the vision statement a number of 'guiding principles' were formulated. Guiding principles are fundamental, enduring tenets that shape M\&S development 
strategies and implementation decisions to realize the above vision. The keywords in these guiding principles are the following:

- cost-effectiveness: better, faster, cheaper

- consistent with "train as you fight" principle

- means to an end, rather than an end in themselves

- allow the linking of live, virtual and constructive

- interoperable and reusable

- exploit the industrial capabilities of Bulgaria

- verified, validated and accredited

- accommodate security needs of NATO and nations

- foster co-operation, respect for national prerogatives

\section{The NATO M\&S Master Plan}

In order to guide the efforts for Bulgaria and to give these efforts a structure, a Master Plan for M\&S is under development. This Master Plan will contain a number of elements. Apart from guidance to use High Level Architecture type language, it provides for educational options and for a management structure.

The Master Plan will outline NATO's effort to co-ordinate, approve and apply costeffectively the collective modeling and simulation capabilities and activities with the Alliance. This plan is expected to evolve as the Alliance and its member nations proceed with its implementation, gain experience and develop additional insights.

Simulations, whether pure software or manned simulators, must also be able to interoperate with various real-world, or "live," systems, such as communications and information systems, weapon systems on instrumented ranges, and system components on test benches. This will facilitate the test and evaluation of the live systems and deliver training, course of action, analysis tools, and mission rehearsal capabilities to humans operating those live systems. NATO will need many different simulations, but for cost-effectiveness, it needs the flexibility to re-use them to the maximum extent, building new representations only when existing simulations cannot meet the need. To get the greatest return on investment, NATO must be able to team these representations and interoperate them in different combinations, called federations, to satisfy its diverse and ever-evolving set of user needs.

NATO should lead the planning and integration of such federations, but leave most of the work on simulation development to the nations. We should exploit our industrial capabilities, reusing and modifying commercial and government simulations before we expend resources to build new systems. 


\section{Objectives}

The M\&S Master Plan contains five objectives. The first objective is to establish a common technical framework to promote the interoperability and reuse of models and simulations between the various users of M\&S. An important achievement here was the relative early adoption of the 'High Level Architecture' as the standard technical architecture for NATO M\&S. This was of particular importance as protracted battles over whose standard is the best would certainly have prevented to achieve the aims set out by NATO. Based upon this choice data interchange standards can be defined.

A second objective of the Master Plan is to provide common services in NATO M\&S. This objective encompasses the compilation of M\&S information, the provision of key education in the field of $M \& S$, the establishment of a Library and of a Help Desk. All in all this is perhaps an ambitious objective, or rather set of subobjectives, but a necessary one in order to start some common NATO infrastructure and common use of M\&S.

The third objective is to develop simulations. This is typically easier stated than realized. In order to achieve real simulation on a NATO level several sub-objectives are identified. Determining priorities and requirements amongst member states and NATO itself is a first such sub-objective. The identification of strategies (existing ones or under development) in the nations is also important in this perspective. Given strategies, requirements and priorities, the next step is the allocation of resources. As funds are typically limited and as their use is often intended as a facilitator for cooperation between member states, the resources available in the nations themselves are by far the more important ones. Having accomplished these sub-objectives, projects can be set up and brought to execution. Lastly, it is of importance to build in appropriate feedback mechanisms on these projects right from the beginning.

A fourth objective, clearly different from the third one, is the use of simulations. The simulations considered under the third objectives are in a certain sense no more than tools. The usefulness of the tools is proven by their application. What has been mentioned on the objective of simulation development is to a large extent also true for the exploitation of simulations. Again planning, prioritizing, the allocation of resources, are important factors. Important also is that those who intend to use these simulation tools provide the necessary databases. Again, after the actual running of the simulation, feedback is essential: has the simulation provided some of the answers looked for, did it provide added value, and so on.

The fifth objective addresses that we live in a dynamic, and not a static world. It is important to monitor and, if appropriate, incorporate technological advances as they become available. Certainly in this field, rich in Information Technology, rapid turnovers of some of the constituting technologies can be expected. Technology 
advances will provide opportunities to increase functional capabilities, performance and overall $M \& S$ effectiveness.

\section{Summary of assessment}

The Steering Group conducted a baseline assessment of the status of M\&S within the Alliance, which provided a high-level overview as of 1997. It is from this baseline that the Alliance moves forward to reach its M\&S vision. The assessment was: "NATO has not yet begun to harness the full potential of M\&S to improve operations. Uses are rich and diverse, but a common Alliance strategy incorporating interoperability and reuse, a key to cost-effectiveness, does not exist yet."

This earlier assessment is still up to date. While start of the M\&S work has been slower than originally intended, it has now become clear that Modeling and Simulation will play an important role in the Alliance's Defense Capability Initiative. Also, M\&S is going to be an important tool in the activities of the NATO Training Group, in particular for the Training and Education Enhancement Program. Computer Aided Exercises will further become more and more common.

Working together as military users, logistic support organizations and technologists will help Bulgaria to make necessary progress in the preparation for NATO integration. This way in the near future Bulgaria will posses increased capability for compatible and more effective planning and operation of its forces.

\section{In the focus of $I \& S$}

In an attempt to cover the broad area of Modeling and Simulation in Defense, we chose the set of articles in the current volume of I\&S.

The first article enables the readers to learn about military simulations and how they are used not only in defense, but also in crisis management in international context. It further updates the reader on the latest developments in the military simulation and training industry. The focus is on the command laboratory simulation but defined in the context of crisis management center concepts.

The next paper describes other interoperability challenges that federation designers and managers face. These challenges relate to the differences between modeling techniques employed by different simulation federates and the logical interpretation of data shared among federates. Using the Federation Development and Execution Process as a guide, the authors identify the key areas where federation developers address simulation interoperability as they apply High Level Architecture to their domains particularly in battle space simulation applications. 
The third paper shows the benefit of using COTS software in the RENAISSANCE efforts, which are construction of reusable, generic, software "building blocks" based on legacy software. In theory, new missions could then select among these building blocks to build systems in an object-oriented manner. The focus is on applications in designing space mission packages.

The first three articles provide examples for the use of simulation on grand strategic and strategic levels. The fourth article in the volume provides an excellent 'tactical' example. It describes distributed simulation as an approach to building large-scale simulation models from a set of independent simulator nodes communicating via a network. The U.S. Army uses distributed simulation systems for both training and analysis. Those systems include both crewed simulators and computer generated forces (CGF) systems; the latter use software, rather than human crews, to generate the behavior of entities in the simulated battlefield. CGF systems must include algorithms for all of the tactical behaviors that are needed for the simulation. One such tactical behavior is "Fire Zone Defense." An algorithm for this behavior must select defensive deployment locations on the terrain for the individual entities (e.g., tanks) of a unit (e.g., a company) to effectively defend an assigned engagement area. The entities of the unit then move to those locations. The paper presents a new algorithm for the behavior combining a geometric terrain analysis algorithm with a greedy optimization algorithm.

The second group of papers presents views and activities of Bulgarian scientists in the area of simulation of moving platforms. The articles examine the main steps in the process of creating a simulator for moving platforms. This principal task includes the following items: understanding the behavior of the simulated object, appropriate mathematical modeling and 3-dimensial visualization. The general features of a fuzzy controlled semi-automatic missile are studied and missile response against the mobile target is evaluated and discussed. The main characteristics of simulator for antitank wire guided missile designed with DirectX7 SDK are presented.

The third group of articles presents the status of modeling and simulation in the Ministry of Defense of Bulgaria and a sample of R\&D activities of Bulgarian military and civilian specialists. This group is intended to underline the necessity of conceptualizing and implementing NATO compatible Bulgarian policy towards M\&S for defense purposes. First, we need to start a discussion on the national problems in Modeling and Simulation. This discussion is expected to involve Bulgarian and NATO experts and to proceed with initial concept implementation, to gain experience and develop additional insight. It is essential that all M\&S experts should devote some time and thought to the consideration of the major problems with which we are currently faced. Some of them can be solved with relative rapidity given common sense and a correctly appreciated self-interest. Others will require foresighted 
planning and patience as, one by one, the necessary steps are taken, leading to readjustment of concepts and inauguration of new attitudes to Modeling and Simulation. The key to current troubles stems not only from the economic difficulties and limited budgets in the last ten years, but more so from the rigid thinking, from the culture of taking without giving, accepting and not sharing, grasping and not disseminating. The goals of the concept are to clarify the real state of Defense Modeling and Simulation in Bulgaria and to establish the initial conditions for creating a Modeling and Simulation Master Plan. Meeting the military requirements will be an evolutionary process as needs are further refined across the spectrum of mission areas. M\&S support will concentrate on activities at the operational and simulator levels. We think that we have one main goal; fortunately or not, we also have many ways to reach this main goal.

The last group of papers is devoted to enabling technologies and education in M\&S design. In the first article, an approach to the next generation televirtual environments that integrate collaboration with distributed computing and modern modeling and simulation technologies is presented. The authors follows the 3-tier architecture with the Web Object (Java/CORBA) based middleware, VRML/Java3D/DirectX based front-ends and JDBC/PSS/OLEDB based back-ends. The design is tested and the integration concepts by prototyping a multi-user authoring and runtime environment to support WebHLA based distributed military simulations. The authors present taxonomy of collaboratory frameworks and integration paradigm, based on the WebFlow system.

In the next paper an idea for distributed designer for automatic control systems is presented. This investigation may be implemented to develop simulators of moving platforms in the group work.

The last paper illustrates some of the interesting concepts that are explored in the course "Military Simulation Techniques \& Technology". This is a 3-day training course designed to teach engineers and project leaders the essential techniques required to design, build, and operate a military simulation system.

As usual, for readers, interested to learn more about foal topic of the I\&S, this volume presents five books, as well as a significant number of Internet addresses.

1. Official NATO and RTB information was used here and further down in this editorial. 